\title{
Early fibroproliferative changes on high-resolution CT predict mortality in COVID-19 pneumonia patients with ARDS
}

\section{Zhilin Zeng}

Department and Institute of Infectious Disease, Tongji Hospital, Tongji Medical College, Huazhong University of Science and Technology,No. 1095, Jie Fang Road, Han Kou District, Wu Han 430030, Hu Bei Province, China https://orcid.org/0000-0003-3195-4010

\section{Min Xiang}

Department of Radiology, Tongji Hospital, Tongji Medical College, Huazhong University of Science and Technology, No. 1095, Jie Fang Road, Han Kou District, Wu Han 430030, Hu Bei Province, China https://orcid.org/0000-0003-1033-6889

\section{Hanxiong Guan}

Department of Radiology, Tongji Hospital, Tongji Medical College, Huazhong University of Science and Technology, No. 1095, Jie Fang Road, Han Kou District, Wu Han 430030, Hu Bei Province, China https://orcid.org/0000-0001-9675-4496

\section{Yiwen liu}

Department of Radiology, Tongji Hospital, Tongji Medical College, Huazhong University of Science and Technology, No. 1095, Jie Fang Road, Han Kou District, Wu Han 430030, Hu Bei Province, China https://orcid.org/0000-0003-0350-6472

\section{huilan Zhang}

Department of Respiratory Medicine, Tongji Hospital of Tongji Medical College, Huazhong University of Science and Technology, No. 1095, Jie Fang Road, Han Kou District, Wu Han 430030, Hu Bei Province, China

\section{Liming Xia}

Department of Radiology, Tongji Hospital, Tongji Medical College, Huazhong University of Science and Technology, No. 1095, Jie Fang Road, Han Kou District, Wu Han 430030, Hu Bei Province, China https://orcid.org/0000-0001-8481-3380

\section{Zhan Juan ( $\nabla$ 809114460@qq.com)}

Department of dermatology, Tongji Hospital, Tongji Medical College, Huazhong University of Science and Technology, No. 1095, Jie Fang Road, Han Kou District, Wu Han 430030, Hu Bei Province, China Qiongjie Hu ( $\sim$ qihu@outlook.com )

Department of Radiology, Tongji Hospital, Tongji Medical College, Huazhong University of Science and Technology, No. 1095, Jie Fang Road, Han Kou District, Wu Han 430030, Hu Bei Province, China 


\section{Research Article}

Keywords: Acute respiratory distress syndrome, fibrosis, COVID-19, SARS-CoV-2

Posted Date: June 3rd, 2020

DOI: https://doi.org/10.21203/rs.3.rs-32245/v1

License: (c) (i) This work is licensed under a Creative Commons Attribution 4.0 International License. Read Full License 


\section{Abstract}

Objectives: To investigate the chest high-resolution CT (HRCT) findings in coronavirus disease 2019 (COVID-19) pneumonia patients with acute respiratory distress syndrome (ARDS) and to evaluate its relationship with clinical outcome.

Materials and Methods In this retrospective study, seventy-nine COVID-19 patients with ARDS were recruited. Clinical data were extracted from electronic medical records and analyzed. HRCT scans, obtained within 3 days before clinical ARDS onset, were evaluated by three independent observers and graded into six findings according to the extent of fibroproliferation. Multivariable Cox proportional hazard regression analysis was used to assess the independent predictive value of the CT score and radiologically fibroproliferation. Patient survival was determined by Kaplan-Meier analysis.

Results: Compared with survivors, non-survivors showed higher of lung fibroproliferation, whereas there no significant differences in the area of increased attenuation without traction bronchiolectasis or bronchiectasis. A HRCT score $<230$ enabled prediction of survival with $73.5 \%$ sensitivity and $93.3 \%$ specificity (AUC $=0.9 ; 95 \% \mathrm{Cl} 0.831$ to 0.968 ). Multivariate Cox proportional hazards model showed that the HRCT score is a significant independent risk factor for mortality (HR 13.007; 95\% Cl 3.935 to 43.001). Kaplan-Meier analysis revealed HRCT score $\geq 230$ was associated with higher fatality rate. Organ injury occurred less frequently in patients with HRCT score $<230$ compared to those with HRCT score $\geq 230$.

Conclusion: Early pulmonary fibroproliferative changes in HRCT predicts increased mortality and susceptibility to organ injury in COVID-19 pneumonia patients with early ARDS.

\section{Key Points}

-Pulmonary fibroproliferation occurs in the early stage of ARDS due to COVID-19 pneumonia, manifested by the areas of traction bronchiolectasis or bronchiectasis within increased attenuation on HRCT scan.

- Compared with survivors, non-survivors showed higher percentage of lung fibroproliferation, whereas there no significant differences in the area of increased attenuation without traction bronchiolectasis or bronchiectasis.

-The extent of fibroproliferative changes on HRCT at diagnosis of ARDS due to COVID-19 pneumonia was an independent predictive factor for death and organ injury.

\section{Introduction}

Coronavirus disease 2019 (COVID-19) is caused by a new coronavirus called severe acute respiratory syndrome coronavirus 2 (SARS-CoV-2), which emerged in China in December 2019[1; 2]. Until April 26, 2020, there were 209 countries and 2,804,796 confirmed COVID-19 cases globally, including over 193,710 deaths[3]. Most cases infected with SARS-CoV-2 have mild symptoms and good prognosis, 
which clinical symptoms are similar to those of the regular human flu. However, similar to severe acute respiratory syndrome (SARS) and Middle East respiratory syndrome (MERS), COVID-19 could also develop to acute respiratory distress syndrome (ARDS), multiple organ failure or even death $[4 ; 5]$.

Mortality from ARDS still remains above 50\% despite use of low tidal volume ventilation and conservative fluid strategies[6]. ARDS is pathologically classified into three stages in which an initial inflammatory injury with protein-rich edema and hemorrhage is followed by fibroproliferation. The fibroproliferative phase of ARDS has traditionally been regarded as a late event[7]. However, previous study found fibroproliferation is initiated early in ARDS and could predict mortality in ARDS patients[8; 9]. In addition, Ichikado et al reported that fibroproliferation changes in HRCT could predict mortality in ARDS patients caused by pneumonia, aspiration, sepsis, etc[10]. Bilateral areas of ground-glass attenuation and airspace consolidation as well as involvement of multiple lung lobes were common chest CT finding in severe and critical COVID-19 pneumonia patients[11]. Recently, Zhang et al observed diffuse alveolar damage (DAD) and alveolar interstitial fibrosis in lung biopsy from a deceased COVID-19 pneumonia patient[12]. To our knowledge, no study has been performed to evaluate chest CT patterns, especially early fibroproliferative changes and their eventual prognosis value in COVID-19 pneumonia patients with ARDS. Thus, in this retrospective study, we evaluate the relation between extent of early fibroproliferative changes in HRCT and outcome in COVID-19 pneumonia with early ARDS.

\section{Materials And Methods}

\section{Study design and participants}

This study was approved by the Institutional Review Board (IRB) of Tongji Hospital, Tongji Medical College, Huazhong University of Science \& Technology (IRB ID: TJ-C0200108). Patient consent in this retrospective study was waived by the Ethics Commission of the designated hospital for emerging infectious diseases. Patients were admitted from January 5 to February 16,2020 . The inclusion criteria were as follows: 1 ) real-time reverse transcription polymerase chain reaction (RT-PCR) assay detection of SARS-CoV-2 nucleic acid positive in throat swabs or nasopharyngeal swab; 2) diagnosis of ARDS using the Berlin definition[13] and interim guidance for clinical management of severe COVID-19 published by World Health Organization (WHO) [14]; 3) CT scan of the chest performed within 3 days before clinical ARDS onset. Exclusion criteria were as follows: pre-existing chronic pulmonary fibrosis and bronchiectasis were strictly excluded by history taking, documented from review of radiological reports and the initial CT imaging data on HRCT scans suggesting. Patients were followed up to April 17, 2020. Finally, seventy-nine patients were enrolled including 45 survivors and 34 non-survivors.

\section{Data collection}

Medical record information including demographic, clinical, laboratory, treatment and outcome data were collected and extracted by using data collection forms.

\section{CT Examination}


All patients underwent whole lung volumetric HRCT scanning of the chest within 0-3 days (median 1) before the onset of ARDS. CT was performed in the supine position during end-inspiration without intravenous contrast medium with various CT scanners using standard-dose chest CT protocols (GE Healthcare, Philips, or Toshiba Medical Systems). Imaging parameters were as follows: $80-120 \mathrm{kVp}$ tube voltage, automated tube current modulation $(60-300 \mathrm{~mA})$, rotate time of $0.5 \mathrm{~s}$, pitch of $0.984: 1$, slice thickness of $1.25 \mathrm{~mm}$, reconstruction matrix: $512 \times 512$, with selected differences according to machine types.

\section{HRCT assessment}

All CT images were reviewed respectively by three radiologists (MX, QJH, HXG with 10, 10 and 20 years of clinical experience, respectively), who were unaware of patient outcome. Disagreements were resolved by consensus. Chest CT images assessed the presence and extent of areas for the following characteristics based on the recommendations in Fleischner Society terminologies and similar studies: ground-glass opacity (GGO)『airspace consolidation, traction bronchiectasis, traction bronchiolectasis, and honeycombing $[15 ; 16]$. When bronchi were irregular in contour or larger than adjacent pulmonary artery, the bronchus within areas of parenchymal abnormality was recognized as traction bronchiectasis. Traction bronchiolectasis was identified by means of the presence of dilated bronchioles within areas with parenchymal abnormality. Honeycombing was defined as small, stacked 2-20 mm cysts in the subpleural lung without intervening lung parenchyma.

\section{Scoring of HRCT Finding}

The HRCT findings were graded on a scale of 1-6 based on the classification by Ichikado and colleagues[10; 16], which was correlating with previously described pathology: score of 1 , normal attenuation; score of 2, ground-glass attenuation; score of 3, consolidation; score of 4 , ground-glass attenuation with traction bronchiolectasis or bronchiectasis; score of 5 , consolidation with traction bronchiolectasis or bronchiectasis; and score of 6, honeycombing. The extent of involvement of each abnormality was assessed independently for each of three zones of each lung: upper (above the carina), middle (below the carina and up to the inferior pulmonary vein), and lower (below the inferior pulmonary vein) zones. The extent of each abnormality was visually estimated to the nearest $10 \%$ of parenchymal involvement in each zone, and then was obtained by average the six zones extent. The abnormality score for each zone was obtained by multiplying the extent of involvement by each grading score (the score of 1-6) and then the total CT score was calculated by adding the averages for each index of the six zones.

\section{Statistical analysis}

All statistical analyses were performed using SPSS 20.0 software. The quantitative data of normal distribution were presented as mean $\pm S D$ (minimum-maximum), and those of abnormal distribution were expressed as median (IQR). Normally distributed variables were compared by using the paired t-test; abnormal distributed variables were compared by using Mann-Whitney U test. The qualitative data were presented as percentage (\%) and analyzed with Fisher's exact test or chi-square test. To analyze the CT 
score as a predictor of survival of ARDS, receiver operator characteristic (ROC) curves and the corresponding area under the curve (AUC) was used to determine the cut-off value of the CT score yielding the highest sensitivity and specificity. Cox proportional hazards regression analysis was used to evaluate the influence of the CT score on survival and radiologically fibroproliferation while adjusting for other prognostic clinical factors, such as age, severity of illness and non-pulmonary organ dysfunctions that had been reported. Patient survival was determined by Kaplan-Meier analysis. For all statistical analyses, $p<0.05$ was considered significant.

\section{Results}

\section{Clinical and Laboratory Findings}

Characteristics, laboratory findings, complications and treatment of patients were showed in Table 1. A total 79 COVID-19 patients with ARDS were enrolled in the research, including 45 survivors and 34 nonsurvivors. The average ages of the survivors and non-survivors were 64.0 and 66.5 years, respectively. The proportion of male patients in survivors was lower than in non-survivors ( $53 \%$ vs. $76 \%, p=0.035)$. The mean SPO2/FiO2 ratio of survivors and non-survivors were 222.0 and 146.7. There were significant differences in ratio of qSOFA $\geq 1$ between two groups ( $67 \%$ vs. $91 \%, p=0.010)$. No significant differences were observed between two groups in any comorbidity including chronic respiratory diseases, hypertension, coronary artery disease, diabetes mellitus, chronic kidney disease, chronic liver disease, cerebrovascular disease and tumor. At onset of ARDS, the deceased cases were more likely to have leukocytosis, lymphopenia and thrombocytopenia. In addition, non-survivors displayed elevated levels of blood urea nitrogen, blood creatinine, prothrombin time, D-dimer, cardiae troponin I, procalcitonin compared with survivor. All complications (respiratory failure, heart injury, liver injury and renal dysfunction) significantly differed between survivors and non-survivors. Most patients received antiviral $(95 \%)$, antibacterial (97\%) and glucocorticoid therapy (90\%). With regard to respiratory support, higher percentages of non-survivors received high oxygen flow, noninvasive ventilation and invasive mechanical ventilation (Table1). 
Table1. Characteristics, laboratory findings, complications and treatment in non-survivors and survivors

\begin{tabular}{|c|c|c|}
\hline $\begin{array}{l}\text { tal } \\
\qquad(\mathrm{n}=79)\end{array}$ & $\begin{array}{l}\text { Survivors } \\
\qquad(\mathrm{n}=45)\end{array}$ & $\begin{array}{c}\text { Non-survivors } \\
(\mathrm{n}=34)\end{array}$ \\
\hline
\end{tabular}

\section{Characteristics}

\begin{tabular}{|c|c|c|c|c|}
\hline Age, yrs & $65.0(57.0-71.0)$ & $64.0(56.5-71.0)$ & $66.5(57.0-73.0)$ & \\
\hline Males, n (\%) & $50(63 \%)$ & $24(53 \%)$ & $26(76 \%)$ & 0.415 \\
\hline $\mathrm{SPO} 2 / \mathrm{FiO} 2$ ratio & $\begin{array}{l}\text { 177.4(134.4- } \\
231.7)\end{array}$ & 222.0(154.1-231.7) & 146.7(90.0-225.6) & 0.035 \\
\hline qSOFA $\geq 1$ & 61 & 30 & 31 & 0.010 \\
\hline \multicolumn{5}{|l|}{ Any comorbidity } \\
\hline $\begin{array}{l}\text { Chronic respiratory } \\
\text { diseases, } n(\%)\end{array}$ & $10(13 \%)$ & $5(11 \%)$ & $5(15 \%)$ & 0.738 \\
\hline Hypertension, n (\%) & $39(49 \%)$ & $26(58 \%)$ & $13(38 \%)$ & 0.085 \\
\hline $\begin{array}{l}\text { Coronary artery disease, } \mathrm{n} \\
\text { (\%) }\end{array}$ & $7(9 \%)$ & $2(4 \%)$ & $5(14 \%)$ & 0.133 \\
\hline Diabetes mellitus, n (\%) & $13(16 \%)$ & $10(22 \%)$ & $3(9 \%)$ & 0.112 \\
\hline $\begin{array}{l}\text { Chronic kidney disease, } \mathrm{n} \\
(\%)\end{array}$ & $3(4 \%)$ & $0(0)$ & $3(8 \%)$ & 0.076 \\
\hline Chronic liver disease, $\mathrm{n}(\%)$ & $3(4 \%)$ & $0(0)$ & $3(9 \%)$ & 0.076 \\
\hline Cerebrovascular disease & $3(4 \%)$ & $1(2 \%)$ & $2(6 \%)$ & 0.574 \\
\hline Tumor, n (\%) & $2(3 \%)$ & $0(0)$ & $2(6 \%)$ & \\
\hline
\end{tabular}

\section{Laboratory findings}

White blood cell count

7.7(5.4-12.1)

$6.5(4.8-10.5)$

8.9(5.5-13.7)

$\times 10^{\wedge} 9 / \mathrm{L}$

$0.7(0.5-1.0)$

$0.5(0.3-0.9)$

0.040

Lymphocyte count, $\times 10^{\wedge} 9 / \mathrm{L}$

0.7(0.4-0.9)

$94.0(146.0-290.0)$

Platelet count, $\times 10^{\wedge} 9 / \mathrm{L}$

$169.0(127.0$

237.0)

Alanine aminotransferase, U/L

27.5(17.0-39.3)

$29.0(17.5-39.0)$

157.0(116.3-196.5)

0.182

Aspartate aminotransferase,

$36.0(25.0-50.5)$

$34.0(24.5-46.5)$

$27.0(17.0-45.0)$

$\mathrm{U} / \mathrm{L}$

$9.0(6.9-12.9) \quad 9.2(7.1-12.6)$

41.0(29.5-67.0)

Total bilirubin, $\mathrm{mmol} / \mathrm{L}$

6.4(5.0-10.0)

5.3(4.1-6.7)

8.4(5.9-14.5)

Blood urea nitrogen, $\mathrm{mmol} / \mathrm{L}$

$76.0(64.0-93.3)$

68(60.5-86.5)

$9.0(6.8-13.2)$

Blood creatinine, $\mu \mathrm{mol} / \mathrm{L}$

14.6(13.9-16.1)

14.3(13.7-15.0)

80.0(72.0-122.0)

Prothrombin time, s

2.2(0.9-10.2)

$2.0(0.7-2.8)$

15.5(14.1-17.9)

D-dimer, $\mu \mathrm{g} / \mathrm{mL}$

11.2(6.2-32.0)

8.5(4.1-20.4)

$4.9(1.3-21.0)$

Cardiae troponin I, pg/mL

23.1(8.4-146.7) 


\section{Complications}

\begin{tabular}{|c|c|c|c|c|}
\hline Respiratory failure, n (\%) & $33(42 \%)$ & $1(2 \%)$ & $32(94 \%)$ & $<0.001$ \\
\hline Heart injury, n (\%) & $18(23 \%)$ & $5(11 \%)$ & 13(38\%) & 0.004 \\
\hline Liver injury, n (\%) & $11(14 \%)$ & $2(4 \%)$ & $9(26 \%)$ & 0.008 \\
\hline Renal dysfunction, n (\%) & $13(16 \%)$ & $3(7 \%)$ & $10(29 \%)$ & 0.007 \\
\hline Treatment & & & & \\
\hline Antibiotics, n (\%) & $77(97 \%)$ & $43(96 \%)$ & $34(100 \%)$ & 0.503 \\
\hline Antivirus, n (\%) & $75(95 \%)$ & $44(98 \%)$ & $31(91 \%)$ & 0.309 \\
\hline Glucocorticoid therapy, n (\%) & $71(90 \%)$ & $40(89 \%)$ & $31(91 \%)$ & 1.000 \\
\hline $\begin{array}{l}\text { Intravenous immunoglobulin, } \mathrm{n} \\
(\%)\end{array}$ & $27(34 \%)$ & $12(27 \%)$ & $15(44 \%)$ & 0.105 \\
\hline High oxygen flow, n (\%) & 15(19\%) & $4(9 \%)$ & $11(32 \%)$ & 0.008 \\
\hline Noninvasive ventilation, $\mathrm{n}(\%)$ & $36(46 \%)$ & $8(18 \%)$ & $28(82 \%)$ & $<0.001$ \\
\hline $\begin{array}{l}\text { Invasive mechanical } \\
\text { ventilation, } \mathrm{n}(\%)\end{array}$ & $13(16 \%)$ & $2(4 \%)$ & $11(32 \%)$ & 0.001 \\
\hline
\end{tabular}

Data are expressed as median (IQR) or $\mathrm{n}(\%)$. Laboratory findings were collected at time of CT examination. $P$ values comparing survivors and Non-survivors are from student $t$ test, Mann-Whitney U test, $\chi \square$ or Fisher's exact test appropriately.

\section{HRCT findings for survivors and non-survivors}

The extent of CT finding in survivors and non-survivors is summarized in Table 2. The average percent lung affected was $75.93 \%$ in non-survivors compared with $59.22 \%$ in survivors $(p<0.001)$. The area of traction bronchiolectasis or bronchiectasis and honeycombing, was indicative of radiologically fibroproliferation. Similarly, the area of traction bronchiolectasis or bronchiectasis significantly smaller in survivors than in non-survivors (Figure 1 and Figure 2), whereas the extent of increased attenuation without traction bronchiolectasis or bronchiectasis did not differ significantly between survivors and nonsurvivors (Table 2). Multivariate Cox proportional hazards model with adjustment for demographic characteristics, severity of illness, non-pulmonary organ dysfunctions and traction bronchiolectasis or bronchiectasis at diagnosis, the total area of the traction bronchiolectasis or bronchiectasis remained an independent risk factor for mortality (HR5.426; $95 \% \mathrm{Cl} 1.307$ to $22.526 ; p=0.020$ ) (Table 3).

Table 2 Extent of each high-resolution CT finding in survivors and non-survivors of ARDS with COVID-19 


\begin{tabular}{|c|c|c|c|}
\hline CT finding & $\begin{array}{l}\text { Survivors } \\
(\mathrm{n}=45)\end{array}$ & $(\mathrm{n}=34)^{\text {Non-survivors }}$ & $\mathrm{p}$ Value \\
\hline $\begin{array}{l}\text { GGO without traction bronchiolectasis } \\
\text { bronchiectasis }\end{array}$ & $34.29(14.28)$ & $32.89(17.69)$ & 0.697 \\
\hline $\begin{array}{l}\text { Consolidation without traction bronchiolectasis } \\
\text { or bronchiectasis }\end{array}$ & $20(12.5-17.08)$ & $16.67(7.92-25.42)$ & 0.168 \\
\hline $\begin{array}{l}\text { Total area without traction bronchiolectasis or } \\
\text { bronchiectasis }\end{array}$ & 54.48(13.2) & $50.74(19.52)$ & 0.312 \\
\hline $\begin{array}{l}\text { GGO with traction bronchiectasis or traction } \\
\text { bronchiolectasis }\end{array}$ & $0(0-2.5)$ & $12.5(2.92-17.08)$ & $<0.001$ \\
\hline $\begin{array}{l}\text { Consolidation with traction bronchiolectasis or } \\
\text { bronchiectasis }\end{array}$ & $0(0-.4 .17)$ & 10.83ロ4.12-18.75凸 & $<0.001$ \\
\hline $\begin{array}{l}\text { Total area with traction bronchiolectasis or } \\
\text { bronchiectasis }\end{array}$ & 1.67(0-6.67) & $\begin{array}{l}21.67 \\
36.67[\end{array}$ & $<0.001$ \\
\hline
\end{tabular}

Data are expressed as median (IQR). $P$ values comparing survivors and non-survivors are by student $t$ test or Mann-Whitney U test appropriately.

Table 3. Multivariate Cox regression analysis of HRCT findings, HRCT score and clinical characteristics associated with mortality in ARDS patients with COVID-19

\begin{tabular}{|c|c|c|c|}
\hline NO & Variable & $P$ Value & HR $(95 \% \mathrm{CI})$ \\
\hline \multirow[t]{2}{*}{1} & HRCT score $\geq 230$ & $<0.001$ & $\begin{array}{l}13.01(3.94- \\
43.00)\end{array}$ \\
\hline & Prothrombin time $\geq 16 \mathrm{~s}$ & 0.028 & $\begin{array}{l}4.76(1.18- \\
19.12)\end{array}$ \\
\hline \multirow[t]{3}{*}{2} & $\begin{array}{l}\text { Existence of GGO with traction bronchiectasis or traction } \\
\text { bronchiolectasis }\end{array}$ & 0.032 & $\begin{array}{l}3.36(1.11- \\
10.20)\end{array}$ \\
\hline & $\begin{array}{l}\text { Existence of consolidation with traction bronchiectasis or traction } \\
\text { bronchiolectasis }\end{array}$ & 0.006 & $\begin{array}{l}4.91(1.58- \\
15.32)\end{array}$ \\
\hline & Prothrombin time $\geq 16 \mathrm{~s}$ & 0.021 & $\begin{array}{l}4.40(1.25- \\
15.52)\end{array}$ \\
\hline \multirow[t]{2}{*}{3} & Total existence of traction bronchiolectasis or bronchiectasis & 0.02 & $\begin{array}{l}5.43(1.31- \\
22.53)\end{array}$ \\
\hline & Prothrombin time $\geq 16 \mathrm{~s}$ & 0.037 & $\begin{array}{l}3.40(1.07- \\
10.76)\end{array}$ \\
\hline
\end{tabular}

Cox proportional hazard regression models were applied to determine the potential risk factors associated with mortality, with the hazards ratio (HR) and 95\% confidence interval(95\%CI) being reported.

\section{Prognostic value of the HRCT score}

The overall HRCT score of survivors (mean, $191.93 \pm 29.47$; range, 146.67-273.33) was significantly lower than that of non-survivors (mean, $255.78 \pm 40.13$; range, 171.67-331.67, $p<0.001$ ). Construction of a ROC curve yielded an optimal cut-off value of a HRCT score of 230 for prediction of survival, with $73.5 \%$ sensitivity and $93.3 \%$ specificity (AUC, $0.9 ; 95 \% \mathrm{Cl} 0.831$ to 0.968 ) (Figure 3 ). Multivariate Cox proportional hazards model analysis, with adjustment for demographic characteristics, general severity, 
underlying disease condition, and non-pulmonary organ dysfunctions, revealed that the HRCT score remained an independent risk factor for mortality (HR 13.007; 95\% Cl 3.935 to 43.001; $p<0.0001$ ) (Table 3). Kaplan-Meier analysis revealed higher CT score was associated with higher fatality rate (Figure 4). However, the overall HRCT scores in 9 non-survivors are less than 230. The non-survivors divided into two groups according to the HRCT score of 230 . The clinical and laboratory findings are compared between the two non-survivor groups. The non-survivors with a lower CT score had the decreased lymphocyte count compared with the non-survivors with a higher CT score $\left(0.9 * 10^{9} / \mathrm{L}\right.$ vs $0.4^{*} 10^{9} / \mathrm{L}, p=0.008$, supplement Table).

\section{Relation between the HRCT score and the number of organ failure}

Organ injury occurred less frequently in patients with HRCT score $<230$ compared to those with HRCT score $\geq 230$ (Table4).

Table 4 Comparison of organ injury between the between higher and lower CT score patients

\begin{tabular}{lcccc}
\hline & Total $(\mathrm{n}=79)$ & CT score $\geq 230$ & $(\mathrm{n}=31)$ & $\begin{array}{c}\text { CT score }<230 \\
(\mathrm{n}=48)\end{array}$ \\
\hline Respiratory failure & $33(42 \%)$ & $23(74 \%)$ & $10(21 \%)$ & $<0.001$ \\
\hline Heart injury & $18(23 \%)$ & $10(32 \%)$ & $8(17 \%)$ & 0.107 \\
\hline Liver injury & $11(14 \%)$ & $8(26 \%)$ & $3(6 \%)$ & 0.014 \\
Renal dysfunction & $13(16 \%)$ & $7(23 \%)$ & $6(13 \%)$ & 0.238 \\
\hline
\end{tabular}

Data are expressed as n (\%). $P$ values comparing higher and lower CT score are from $\chi \square$ or Fisher's exact test appropriately.

\section{Discussion}

In this retrospective cohort study, we comprehensively evaluated and analyzed the HRCT imaging characteristics of 79 COVID-19 pneumonia patients with ARDS. We found that pulmonary fibroproliferation occurs in the early stage of ARDS due to COVID-19 pneumonia, manifested by the areas of traction bronchiolectasis or bronchiectasis within increased attenuation on HRCT scan. Furthermore, we demonstrated the extent of fibroproliferative changes on HRCT and a higher CT score at diagnosis of ARDS due to COVID-19 pneumonia was an independent predictive factor for death. Our observation suggested pulmonary fibroproliferation at the early stage of COVID-19 ARDS is an important determinant of outcome. To our knowledge, it is the first study in which complementary fibroproliferative changes on HRCT as higher HRCT score and to evaluate whether fibroproliferation on HRCT in COVID-19 pneumonia patients with ARDS predict mortality.

Traditionally, ARDS is divided into three stages in which an initial inflammatory phase is followed by fibroproliferation[7]. Interesting, fibroproliferative pathways are activated early in ARDS, demonstrated by increased fibrotic marker in bronchoalveolar lavage fluid (BALF) [9]. HRCT scans of acute interstitial 
pneumonia showed more extensive areas of increased attenuation associated with traction bronchiectasis, which corresponded to fibroproliferative phases of DAD[17]. In the present study, traction bronchiolectasis or bronchiectasis within areas of increased attenuation, suggesting radiologically fibroproliferation, was already detectable on HRCT scans obtained within three days (median 1 day) before the ARDS diagnosis in 49 patients (62.03\%). Some investigations have shown that the typical findings of chest CT images in COVID pneumonia patients are bilateral multiple lobular and subsegmental areas of consolidation and ground-glass opacity, most commonly in the peripheral, subpleural area, or distributed diffusively[18-20]. In addition, Li et al reported that HRCT findings associated with severe and critical COVID-19 pneumonia were bilateral areas of ground-glass attenuation and consolidation in multiple lung lobes. However, this new radiological evidence provided an innovative new method compared with previous investigations. Nevertheless, our study together with previous observations, suggests an alternative to traditional models of the lung injury response, whereby inflammatory and repair mechanisms occur in parallel rather than in series.

Several retrospective cohort studies have clarified clinical risk factors for death in patients with COVID-19 pneumonia[21-24]. However, as we known, few studies have focused on the chest CT imaging appearance and COVID-19 pneumonia mortality. Our data suggested extensive fibroproliferative changes on HRCT in the early stage were associated with ARDS mortality in patients with COVID-19 pneumonia. Similarly, Ichikado et al. showed higher mortality in patients with areas of increased lung attenuation and varicoid bronchiectasis in the setting of clinically diagnosed ARDS caused by diverse diseases[10; 16]. These findings suggest that fibroproliferative changes on HRCT could be used for the assessment of direct and indirect ARDS severity.

The potential mechanisms by which fibroproliferative changes on HRCT might lead to poorer outcome are unclear and are related to pathological process. ARDS patients, who were in the acute exudative phase histologically had a better prognosis than did those who were in the fibroproliferative phase which confirmed by lung biopsy[9]. On the basis of our evaluation of 79 COVID-19 pneumonia ARDS patients, some patients with fibroproliferative changes would be diagnosed with early ARDS if only the parameter of time elapsed since onset of ARDS. Our present data suggest the discrepancy between "clinically" early phase of ARDS and "pathologically" early phase of ARDS. However, it is difficult to distinguish the transition from exudative to fibroproliferative these pathological phases without a lung biopsy in ARDS patients. Chest CT scan could be useful to distinguish these pathological phases and for the early diagnosis of lung injury.

A definitive role of corticosteroids in the treatment of ARDS is not established, however, corticosteroids treatment started after the onset of ARDS was suggested[25]. A recent study showed corticosteroid treatment may be beneficial for COVID-19 pneumonia who have developed ARDS on disease progression[26]. In our study, COVID-19 ARDS patients with limited lung fibroproliferation benefited from corticosteroid treatment. Our results confirmed the hypothesis that the early use of corticosteroid in ARDS patients may improve mortality. Prospective evaluation of HRCT findings in ARDS patients not only has 
its prognostic implications, but also helps therapeutic implications based on the extent of fibroproliferative changes.

In the present study, patients with extensive fibroproliferation shown as higher HRCT score predicts increased mortality with $93.3 \%$ specificity and $73.5 \%$ sensitivity. However, we found that the overall HRCT score in 9 non-survivors was less than 230. We further compared the clinical and laboratory findings between the two non-survivor groups according to the HRCT score of 230. The non-survivors with a lower CT score had the increased lymphocyte count compared with the non-survivors with a higher CT score. Our findings support that suggesting there are subphenotypes of COVID-19 ARDS that affect clinical outcomes.

\section{Abbreviations}

HRCT: high resolution computed tomograph

ARDS: acute respiratory distress syndrome

COVID-19: Corona Virus disease 2019

SARS-Cov-2: Severe acute respiratory syndrome coronavirus 2

ROC: receiver operator characteristic curves

AUC: area under the curve

\section{Declarations}

\section{Acknowledgments}

We express our gratitude to Professor David A. Lynch (the Department of Radiology, National Jewish Health, Denver) and Qihang Chen (Department of Radiology, Beijing Hospital) for consultation on CT images. We are indebted to the frontline medical and nursing staff in Wuhan city who demonstrated selfless and heroic devotion to duty in the face of this COVID-19 outbreak despite the potential threat to their own lives and those of their family members.

\section{Author contributions}

ZLZ and MX contributed equally to this paper. JZ and QJH designed the study, had full access to all data in the study, and take responsibility for the integrity and accuracy of the data analysis. JMZ, YWL, HXG and LMX contributed to patient recruitment, data collection, data analysis, data interpretation and literature search. ZLZ and MX drafted the manuscript. JZ and QJH final revised the manuscript. All authors contributed to data acquisition, data analysis, or data interpretation, and all reviewed and approved the final version of the manuscript. 


\section{Competing interests}

The authors declare no competing interests.

\section{References}

1 Guan W-J, Ni Z-Y, Hu Y et al (2020) Clinical Characteristics of Coronavirus Disease 2019 in China. N Engl J Med. 10.1056/NEJMoa2002032:10.1056/NEJMoa2002032

$2 \quad$ Huang C, Wang Y, Li X et al (2020) Clinical features of patients infected with 2019 novel coronavirus in Wuhan, China. Lancet (London, England). 10.1016/S0140-6736(20)30183-5:S01406736(0120)30183-30185

3 (2020) Coronavirus disease (COVID-2019) situation reports-97. Available via https://www.who.int/docs/default-source/coronaviruse/situation-reports/20200426-sitrep-97-covid19.pdf?sfvrsn=d1c3e800_6

4 Chen $\mathrm{T}, \mathrm{Wu} \mathrm{D}$, Chen $\mathrm{H}$ et al (2020) Clinical characteristics of 113 deceased patients with coronavirus disease 2019: retrospective study. BMJ 368:m1091

5 Zhou F, Yu T, Du R et al (2020) Clinical course and risk factors for mortality of adult inpatients with COVID-19 in Wuhan, China: a retrospective cohort study. Lancet (London, England). 10.1016/s01406736(20)30566-3

6 Fan E, Brodie D, Slutsky AS (2018) Acute Respiratory Distress Syndrome: Advances in Diagnosis and Treatment. Jama 319:698-710

$7 \quad$ Meduri GU (1996) The role of the host defence response in the progression and outcome of ARDS: pathophysiological correlations and response to glucocorticoid treatment. The European respiratory journal 9:2650-2670

8 Meduri GU, Tolley EA, Chinn A, Stentz F, Postlethwaite A (1998) Procollagen types I and III aminoterminal propeptide levels during acute respiratory distress syndrome and in response to methylprednisolone treatment. Am J Respir Crit Care Med 158:1432-1441

9 Marshall RP, Bellingan G, Webb S et al (2000) Fibroproliferation occurs early in the acute respiratory distress syndrome and impacts on outcome. Am J Respir Crit Care Med 162:1783-1788

$10 \mathrm{~K} \mathrm{I}, \mathrm{H} \mathrm{M}, \mathrm{Y} \mathrm{G}$ et al (2012) Fibroproliferative changes on high-resolution CT in the acute respiratory distress syndrome predict mortality and ventilator dependency: a prospective observational cohort study. BMJ open 2:e000545

11 Li K, Wu J, Wu F et al (2020) The Clinical and Chest CT Features Associated with Severe and Critical COVID-19 Pneumonia. Invest Radiol. 10.1097/rli.0000000000000672 
13 Ranieri VM, Rubenfeld GD, Thompson BT et al (2012) Acute respiratory distress syndrome: the Berlin Definition. Jama 307:2526-2533

14 World Health Organization. (2020). Clinical management of severe acute respiratory infection (SARI) when COVID-19 disease is suspected: interim guidance, 13 March 2020. World Health Organization. Available via https://apps.who.int/iris/handle/10665/331446

15 Hansell DM, Bankier AA, MacMahon H, McLoud TC, Müller NL, Remy J (2008) Fleischner Society: glossary of terms for thoracic imaging. Radiology 246:697-722

16 Ichikado K, Suga M, Muranaka $\mathrm{H}$ et al (2006) Prediction of prognosis for acute respiratory distress syndrome with thin-section CT: validation in 44 cases. Radiology 238:321-329

17 Ichikado K, Suga M, Müller NL et al (2002) Acute interstitial pneumonia: comparison of highresolution computed tomography findings between survivors and nonsurvivors. Am J Respir Crit Care Med 165:1551-1556

18 M C, A B, X M et al (2020) CT Imaging Features of 2019 Novel Coronavirus (2019-nCoV). Radiology 295:202-207

$19 \mathrm{XX}, \mathrm{C}$ Y, J Q et al (2020) Imaging and clinical features of patients with 2019 novel coronavirus SARS-CoV-2. European journal of nuclear medicine and molecular imaging 47:1275-1280

20 Y P, H G, S Z et al (2020) Initial CT findings and temporal changes in patients with the novel coronavirus pneumonia (2019-nCoV): a study of 63 patients in Wuhan, China. Eur Radiol. $10.1007 / \mathrm{s} 00330-020-06731-x$

21 Booth CM, Matukas LM, Tomlinson GA et al (2003) Clinical features and short-term outcomes of 144 patients with SARS in the greater Toronto area. Jama 289:2801-2809

22 Choi KW, Chau TN, Tsang 0 et al (2003) Outcomes and prognostic factors in 267 patients with severe acute respiratory syndrome in Hong Kong. Annals of internal medicine 139:715-723

23 Du R-H, Liang L-R, Yang C-Q et al (2020) Predictors of Mortality for Patients with COVID-19 Pneumonia Caused by SARS-CoV-2: A Prospective Cohort Study. European Respiratory Journal. 10.1183/13993003.00524-2020:2000524

24 Li X, Xu S, Yu M et al (2020) Risk factors for severity and mortality in adult COVID-19 inpatients in Wuhan. J Allergy Clin Immunol. 10.1016/j.jaci.2020.04.006 
25 Peter JV, John P, Graham PL, Moran JL, George IA, Bersten A (2008) Corticosteroids in the prevention and treatment of acute respiratory distress syndrome (ARDS) in adults: meta-analysis. BMJ 336:1006-1009

26 Wu C, Chen X, Cai Y et al (2020) Risk Factors Associated With Acute Respiratory Distress

Syndrome and Death in Patients With Coronavirus Disease 2019 Pneumonia in Wuhan, China. JAMA internal medicine. 10.1001/jamainternmed.2020.0994:10.1001/jamainternmed.2020.0994

\section{Figures}



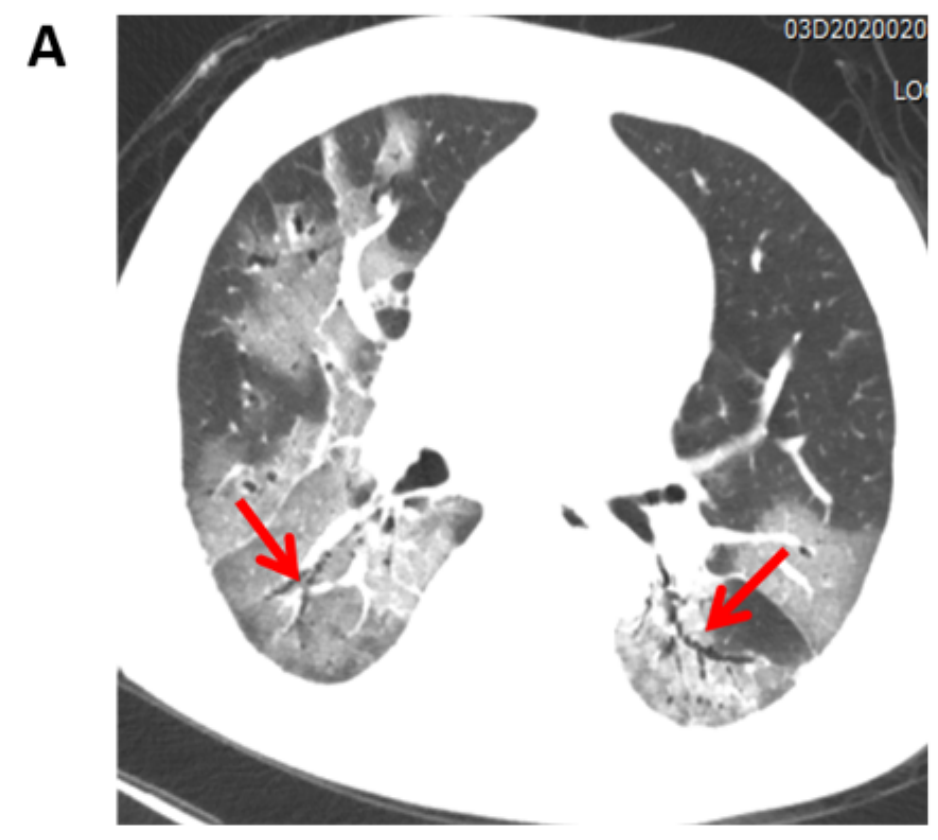

B
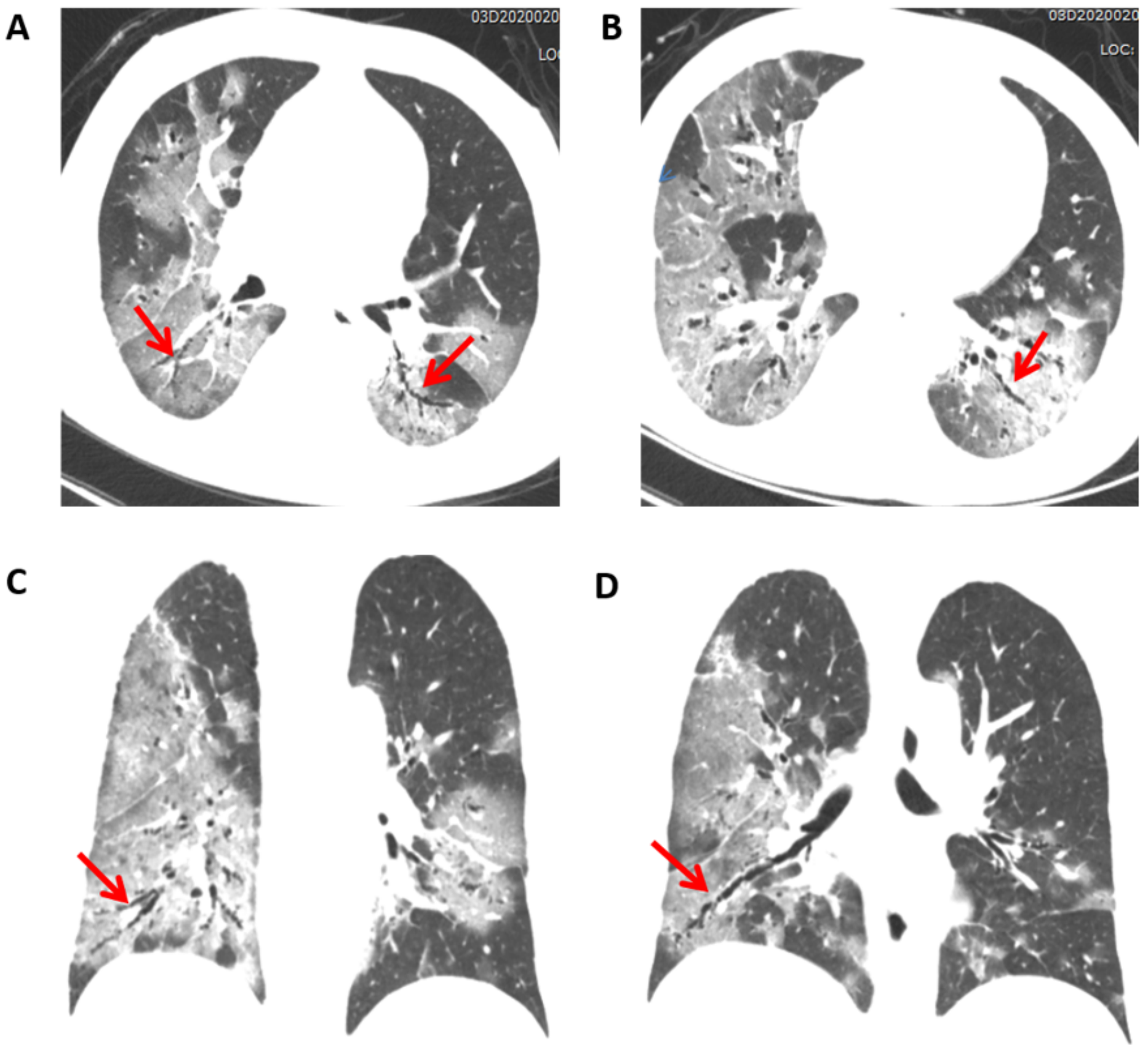

D

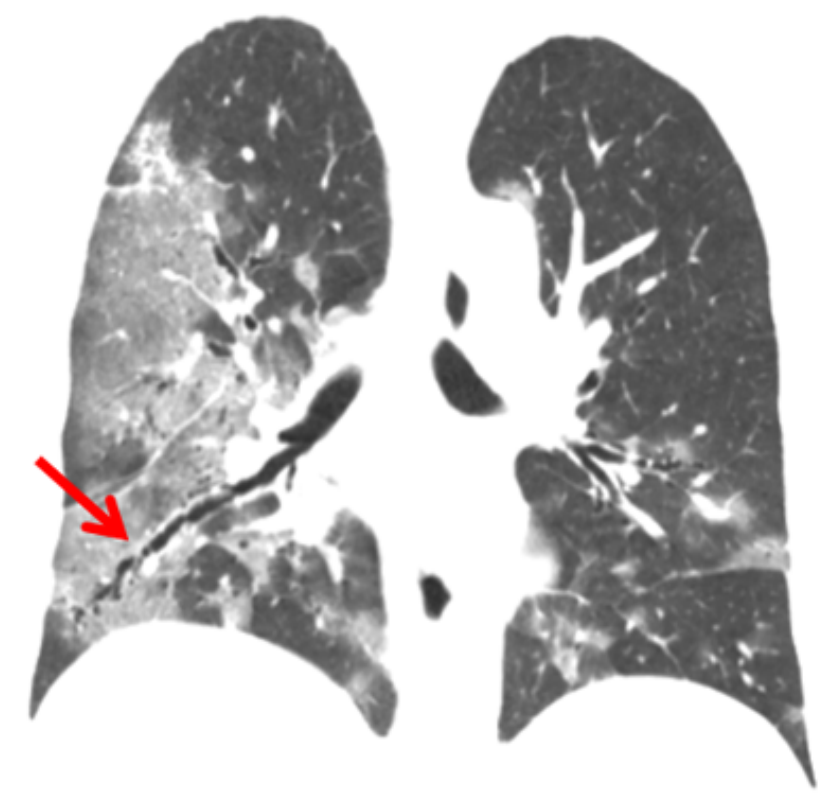

Figure 1

HRCT findings in a 55-year-old man with ARDS due to COVID-19 pneumonia who did not survive. HRCT scan shows bilateral areas of extensive ground-glass opacities associated with traction bronchiolectasis and bronchiectasis (arrows) and small consolidation in most lobes. (A) (B) axial images, (C) (D) coronal images. 
A

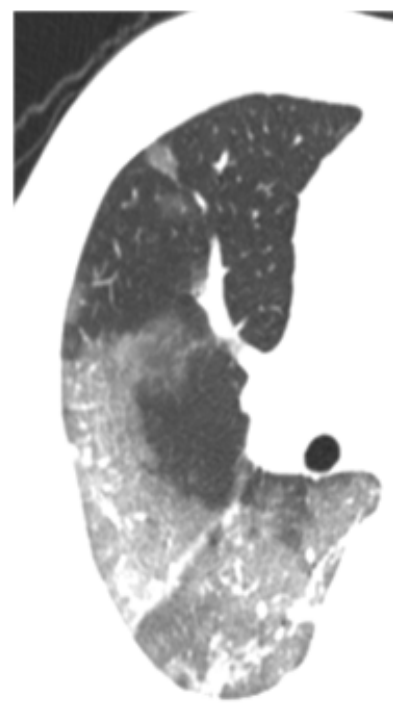

21

62

\section{C}

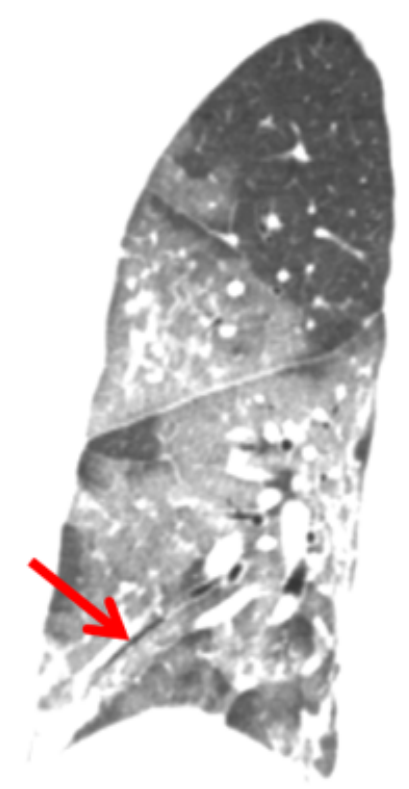

B
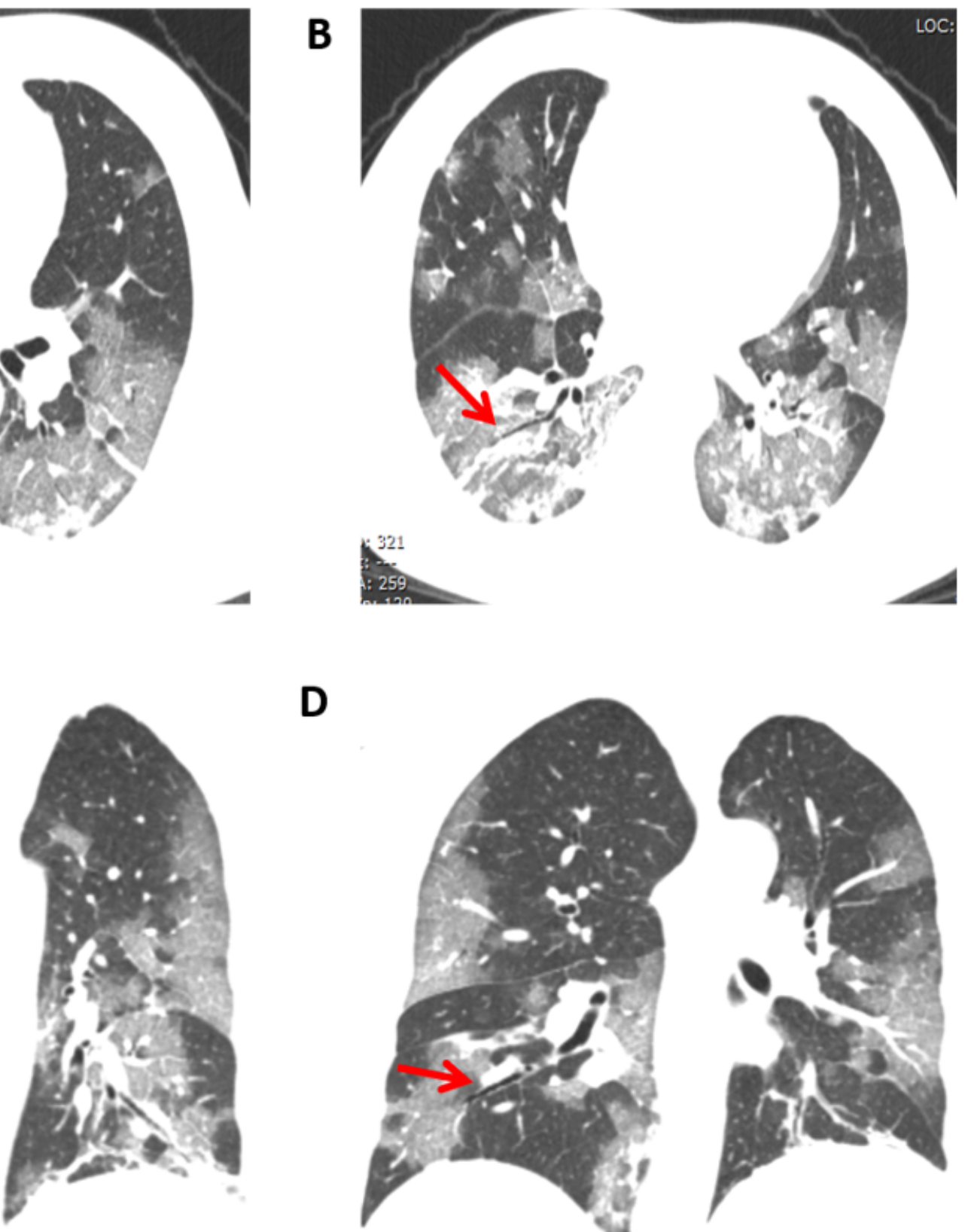

D

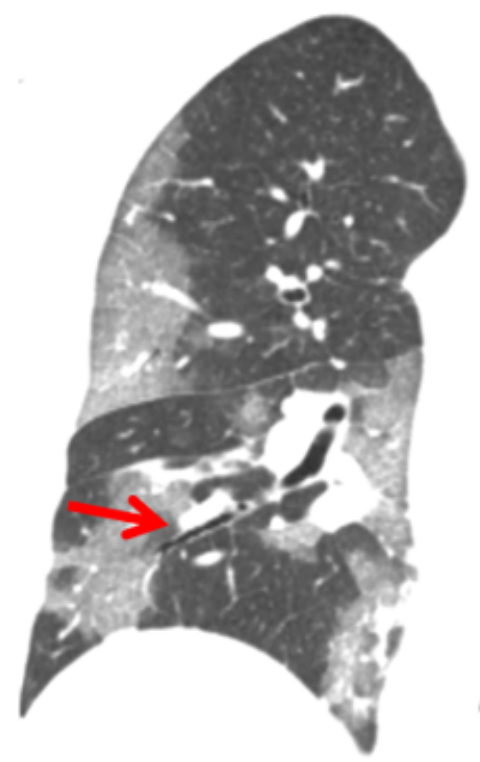

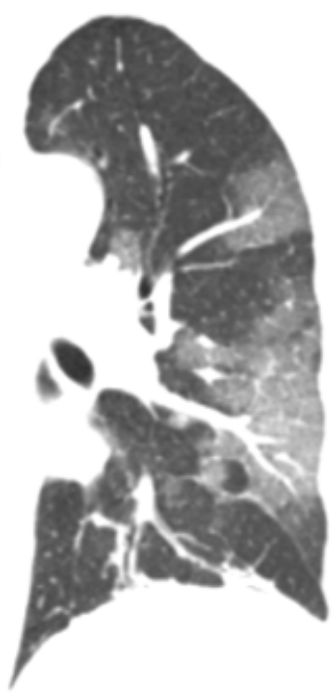

Figure 2

HRCT findings in 40-year-old man with ARDS due to COVID-19 pneumonia who survived. HRCT scan shows extensive ground-glass opacity predominantly and patchy consolidation without traction bronchiolectasis or bronchiectasis (arrows) involved multiple lung lobes. (A) (B) axial images, (C) (D) coronal images. 


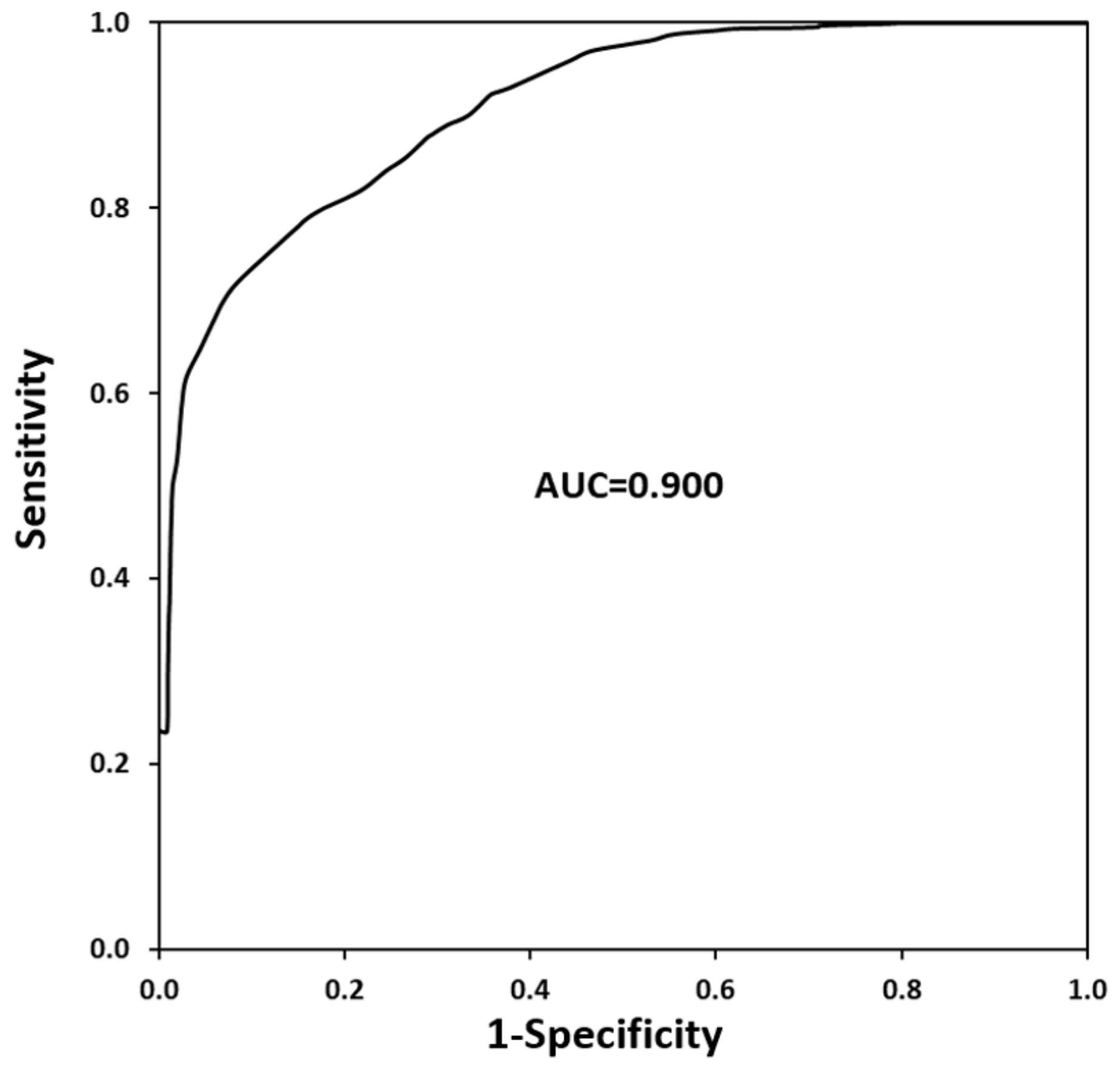

Figure 3

ROC curve of the CT score identified the optimal cut-off value of 230 for prediction of survival. 


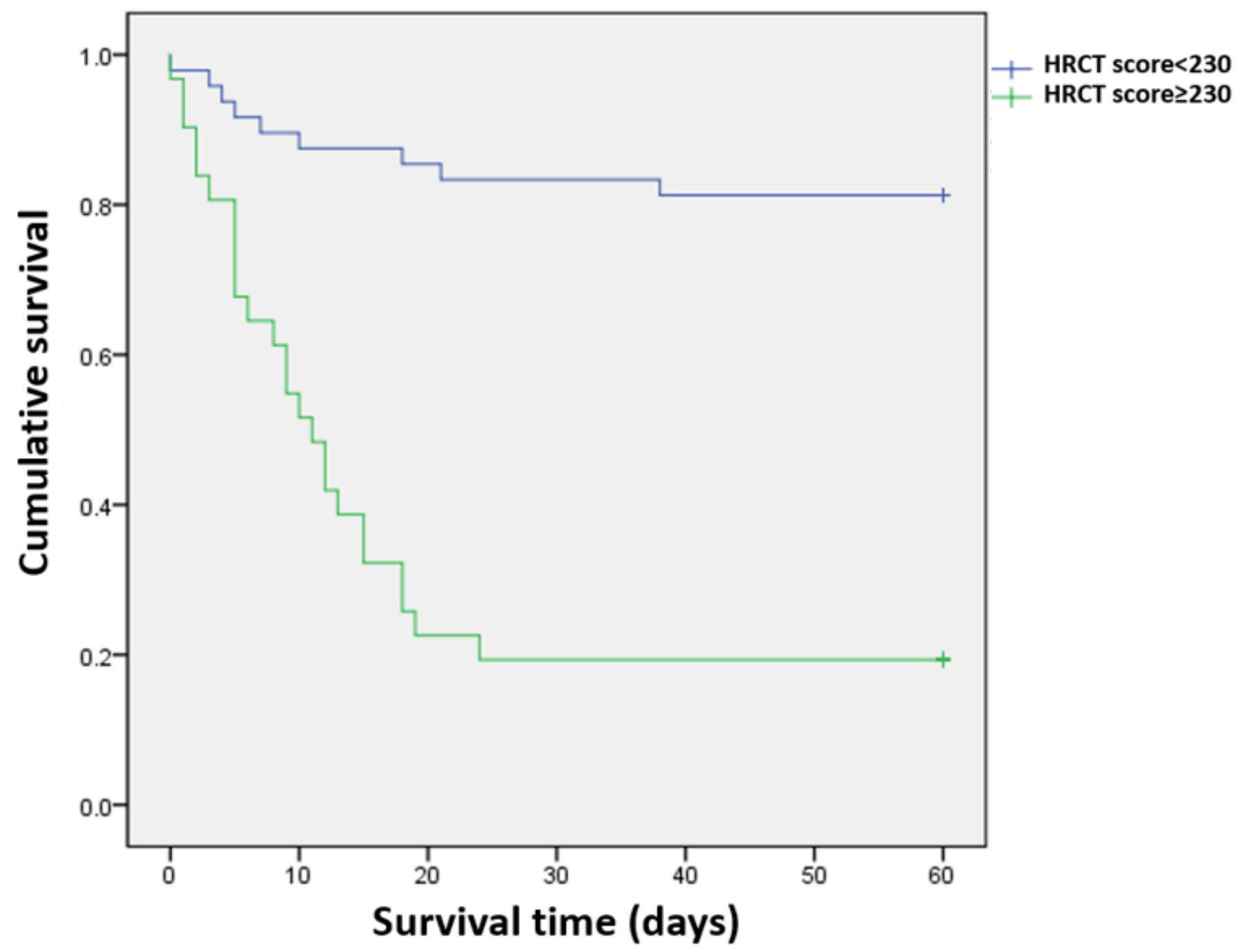

Figure 4

Kaplan-Meier analysis showed a significant difference for the survival ratio for a CT score cut-off of 230 .

\section{Supplementary Files}

This is a list of supplementary files associated with this preprint. Click to download.

- Supplementarytable.docx 